\title{
Adsorption Transition of a Polyelectrolyte on a High-dielectric Charged Substrate
}

\author{
Chi-Ho Cheng* and Pik-Yin Lai \\ Department of Physics and Center for Complex Systems, \\ National Central University, Taiwan 320, Republic of China.
}

(Dated: November 21, 2018)

\begin{abstract}
The behavior of a polyelectrolyte adsorbed on a charged surface of high-dielectric constant is studied by both Monte-Carlo simulation and analytical methods. It is found that in a low ionic strength medium, the transition is first-order with the repulsive charged surface. The surface monomer density, which is the order parameter of the adsorption transition, follows a linear relation with surface charge density. It indicates the polyelectrolyte is compressed on the substrate without any conformational change before the de-sorption. Finally, a new scaling law for the layer thickness is derived and verified by simulation.

PACS numbers: $61.25 . \mathrm{Hq}, 82.35 . \mathrm{Gh}$
\end{abstract}

Polymer adsorption on an attractive surface has drawn considerable interest due to its relation to surface effects in critical phenomena and practical importance in material science and biophysics. It is well established that the adsorption transition is continuous if its attraction on the surface is short-ranged [1, 2, 3, 4]. On the other hand, long-ranged electrostatic interactions in polyelectrolyte systems pose many challenging theoretical problems. Recently the macroion adsorption on an electrostatically attractive interface and the associated charge inversion phenomena of adsorbed polyelectrolytes acquire lots of attention [5, 6, 7].

Previous analytical approaches using the Edwards equation imposed the continuity of the monomer density across the surface and setting the monomer density to zero at the surface $[8,9]$. Within the framework of the self-consistent field method, both the Poisson-Boltzmann equation and Edwards equation were solved simultaneously 10, 11, 12] with zero monomer density at the surface. These treatment however cannot faithfully respect the electrostatic boundary condition. As a result, the adsorption transition would still be continuous whatever the surface potential looks like.
Recently, the theoretical interest to the problem is due to its importance for multi-layer polyelectrolyte adsorption 13, 14, 15. It also raises the question of applying Poisson-Boltzmann theory to polyelectrolyte adsorption because the theory fails to capture the correlation effects.

In this letter, we study the adsorption of a single polyelectrolyte on a high-dielectric substrate in which the image charge attraction is strong. At low ionic strength, the adsorption transition occurs when the surface charges are repulsive instead of the attractive case that were usually studied. The problem is tackled by performing MonteCarlo simulations and also by analytical methods in polymer physics taking full account of the appropriate boundary conditions. It is found that the order of the adsorption transition, the physical mechanism, and the scaling behavior are all different from those of the attractive surfaces.

A polyelectrolyte carrying positive charges is immersed in a medium $(z>0)$ of dielectric constant $\epsilon$. At $z=0$ there is an impenetrable surface of uniform surface charge density $\sigma$. Below the surface $(z<0)$, it is a substrate of dielectric constant $\epsilon^{\prime}$. Denote the charge on a polymer segment $d s$ by $q_{0} d s$, the Hamiltonian is

$$
\begin{aligned}
\mathcal{H}= & \frac{3 k_{\mathrm{B}} T}{2 l_{0}^{2}} \int_{0}^{N} d s\left(\frac{\partial \vec{r}(s)}{\partial s}\right)^{2}+\frac{1}{2} \int_{0}^{N} d s \int_{0}^{N} d s^{\prime}\left(\Gamma \frac{\mathrm{e}^{-\kappa\left|\vec{r}(s)-\vec{r}\left(s^{\prime}\right)\right|}}{\left|\vec{r}(s)-\vec{r}\left(s^{\prime}\right)\right|}-\Gamma^{\prime}\left(2-\delta_{s, s^{\prime}}\right) \frac{\mathrm{e}^{-\kappa\left|\vec{r}(s)-\vec{r}^{\prime}\left(s^{\prime}\right)\right|}}{\left|\vec{r}(s)-\vec{r}^{\prime}\left(s^{\prime}\right)\right|}\right) \\
& +h \int_{0}^{N} d s \kappa^{-1} \mathrm{e}^{-\kappa \vec{r}(s) \cdot \hat{z}}+\omega \int_{0}^{N} d s \int_{0}^{N} d s^{\prime} \delta\left(\vec{r}(s)-\vec{r}\left(s^{\prime}\right)\right)
\end{aligned}
$$

where $s$ is the variable to parametrize the chain, $l_{0}$ the bare persistence length, and $\kappa^{-1}$ the Debye screening length. $\vec{r}(s)=(x(s), y(s), z(s)), \vec{r}^{\prime}\left(s^{\prime}\right)=$ $\left(x\left(s^{\prime}\right), y\left(s^{\prime}\right),-z\left(s^{\prime}\right)\right)$ are the positions of the monomers and their electrostatic images, respectively. $\Gamma=q_{0}^{2} / \epsilon$, $\Gamma^{\prime}=\Gamma\left(\epsilon^{\prime}-\epsilon\right) /\left(\epsilon^{\prime}+\epsilon\right)$, and $h=4 \pi q_{0} \sigma /\left(\epsilon^{\prime}+\epsilon\right)$ are the coupling parameters governing the strength of Coulomb interactions among the monomers themselves, between the polymer and its image, and between the polymer and the charged surface, respectively. The last term in Eq.(1) represents the excluded volume interactions with $\omega>0$ (good solvent regime) in this study. We shall focus on the 
case of a charge polymer in a low ionic strength medium.

The above continuum model is discretized to perform Monte-Carlo simulation. The continuous curve $\vec{r}(s)$ is replaced by a chain of beads $\vec{r}_{i}(i=1, \ldots, N)$ with hardcore excluded volume of finite radius $a$. Total lengths up to $N=120$ are employed. Units of length and energy are set to be $2 a$ and $q_{0}^{2} / 2 \epsilon a$, respectively. Dielectric ratios $\epsilon^{\prime} / \epsilon$ are chosen from 2 to 12.5 (aqueous solution with a metallic substrate). Runs up to $10^{9} \mathrm{MC}$ steps are performed to achieve good statistics.

The adsorption layer can be characterized by the normalized monomer density $\rho(z) . \rho_{a} \equiv \rho(a)$ representing the fraction of monomers being adsorbed on the substrate is chosen as an order parameter to describe the adsorption transition. $\rho_{a}>0$ and $\rho_{a}=0$ characterize the adsorbed and de-sorbed states respectively. In Fig 1 $\rho_{a}$ as a function of the surface charge density $\sigma$ for various $\epsilon^{\prime} / \epsilon>1$ is shown. The discontinuous jump of $\rho_{a}$ across the threshold indicates the transition is first order. We also verified that the energy jump (latent heat) across the transition is proportional to $N$. Similar results were obtained for larger $\kappa^{-1}$.

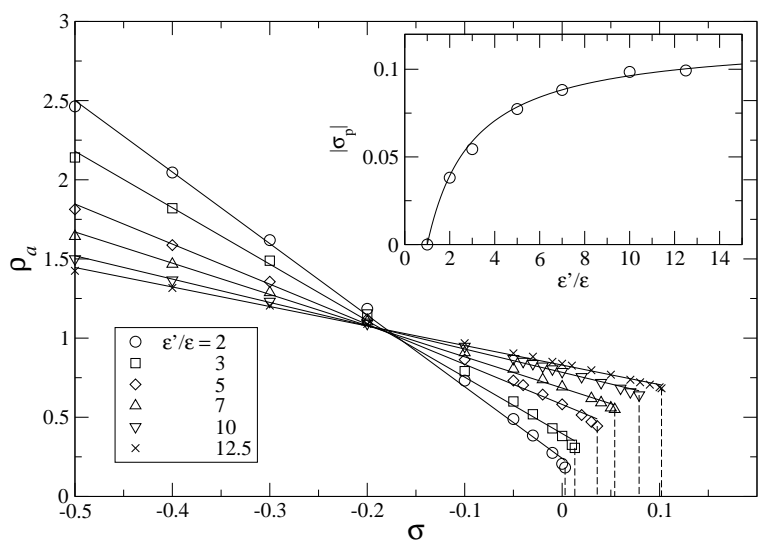

FIG. 1: Monte-Carlo results for the normalized monomer density at the surface, $\rho_{a}$, as a function of surface charge density $\sigma$ (in unit of $q_{0} / 4 a^{2}$ ) for different $\epsilon^{\prime} / \epsilon$ at $\kappa^{-1}=25$. The fitted straight lines are terminated at their adsorption transition points. The vertical dashed lines are drawn as guides to the eyes. Inset: The polarization surface charge density induced by the polyelectrolyte, $\left|\sigma_{\mathrm{p}}\right|$, as a function of dielectric ratio $\epsilon^{\prime} / \epsilon$. The sign of $\sigma_{\mathrm{p}}$ is opposite to $q_{0}$ and is negative. The solid curve is fitted from Eq.(6) with $\sigma_{\text {poly }}=0.118$.

Furthermore, the data in Fig 1 also indicate that $\rho_{a}$ is linear in $\sigma$ with the slope depending on the ratio of $\epsilon^{\prime} / \epsilon$. Such a linear relation between $\rho_{a}$ and $\sigma$ can be understood from the electrostatic boundary conditions that the system has to satisfy. The electric potential $\phi(z)$ in the neighborhood of the $z=0$ boundary obeys,

$$
-\left.\frac{\partial \phi}{\partial z}\right|_{z=0^{+}}+\left.\frac{\partial \phi}{\partial z}\right|_{z=0^{-}}=-\frac{4 \pi}{\epsilon}\left(\frac{2 \sigma}{\epsilon^{\prime} / \epsilon+1}+\sigma_{\mathrm{p}}\right)
$$

where $\sigma_{\mathrm{p}}$ is the polarization surface charge density induced by the polymer only, which depends on $\epsilon^{\prime} / \epsilon$ but is independent of $\sigma$ in the adsorbed regime near the transition. Notice that $\sigma_{\mathrm{p}}$ in general is a complicated function since it relies on the polymer conformation. Also, if one treats the polymer as a marcomolecule with a welldefined surface, its surface charge density at $z=a$ should be proportional to the monomer density $\rho_{a}$, this also applies to the electric field in the $z<0$ region,

$$
\begin{aligned}
& \left.K \frac{\partial \phi}{\partial z}\right|_{z=a^{-}}=-\frac{4 \pi}{\epsilon} \rho_{a} \\
& \left.K \frac{\partial \phi}{\partial z}\right|_{z=0^{-}}=-\frac{4 \pi}{\epsilon} \frac{2 \epsilon^{\prime}}{\epsilon^{\prime}+\epsilon} \rho_{a}
\end{aligned}
$$

where $K>0$ is the corresponding proportional constant. Applying the continuity condition for the electric field from $z=0^{+}$to $z=a^{-}$, and using Eqs.(2)-(41), one gets the linear behavior

$$
\rho_{a}=-\frac{2 K}{\epsilon^{\prime} / \epsilon-1}\left(\sigma+\frac{\epsilon^{\prime} / \epsilon+1}{2} \sigma_{\mathrm{p}}\right) .
$$

Notice that $K$ and $\sigma_{\mathrm{p}}$ are functions of $\epsilon^{\prime} / \epsilon$. The linear behavior in Eq. (5) is confirmed by the simulation data as shown in Fig 1 the slope decreases monotonically with $\epsilon^{\prime} / \epsilon$. Substituting $\sigma=0$ into Eq.(5), we get the polarization surface charge density as a function of dielectric constant ratio,

$$
\sigma_{\mathrm{p}}=-\frac{\left.\rho_{a}\right|_{\sigma=0}}{K} \frac{\epsilon^{\prime}-\epsilon}{\epsilon^{\prime}+\epsilon} .
$$

$K$ for different dielectric ratios is obtained from the slopes of different straight lines presented in Fig.1. $\sigma_{\mathrm{p}}$ as a function of $\epsilon^{\prime} / \epsilon$ is then fully determined from simulation data as shown in the inset of Fig.1. It fits very well to Eq.(6) with $\left.\sigma_{\text {poly }} \equiv \rho_{a}\right|_{\sigma=0} / K=0.118$. It suggests the conformation of the adsorbed polymer is compressed on the high-dielectric substrate. We have also check the adsorption layer thickness is independent of the number of monomer $N$, which is consistent with the picture of a compressed state. There is no conformational change during the de-sorption contrary to the scaling results as predicted by Borisov et.al. [19] for the attractive surface.

The polyelectrolyte behaves as electric blobs arranged longitudinally and lie down parallel to the surface. Increasing the attraction from the charged surface reduces the $z$-fluctuation amplitude of the chain, but the effective in-plane surface charge distribution of the polyelectrolyte does not change. Hence the polarization $\sigma_{\mathrm{p}}$ is independent of $\sigma$. The excluded volume effect is safely ignored because it takes almost no effect in the $z$-direction. The effect from self-electrostatic interaction of the polyelectrolyte can be absorbed into the bare persistence length from $l_{0}$ to $l$.

Because the monomer would feel the strongest attraction from its direct image around the adsorption regime, 
the $\Gamma^{\prime}$-term in Eq. (11) is approximated by the interaction of each monomer and its corresponding image only. The residual attraction from the images of other monomers is then adsorbed into the coupling parameter $\Gamma^{\prime}$ from $q_{0}$ to $q$. The partition function is reduced to

$$
\begin{aligned}
Z=\int & \mathcal{D}[\vec{r}(s)] \exp \left[\int _ { 0 } ^ { N } d s \left\{-\frac{3}{2 l^{2}}\left(\frac{\partial \vec{r}(s)}{\partial s}\right)^{2}\right.\right. \\
& \left.\left.+\frac{\beta \Gamma^{\prime}}{4} \frac{\mathrm{e}^{-2 \kappa \vec{r}(s) \cdot \hat{z}}}{\vec{r}(s) \cdot \hat{z}}-\beta h \kappa^{-1} \mathrm{e}^{-\kappa \vec{r}(s) \cdot \hat{z}}\right\}\right] .
\end{aligned}
$$

Transforming the variable from $\vec{r}(s)$ to the normalized monomer density $\rho(\vec{r})=\frac{1}{N} \int_{0}^{N} d s \delta(\vec{r}-\vec{r}(s))$ by introducing an auxiliary field, then applying the ground state dominance approximation in large- $N$ limit and by variational principle [17, 18], one obtains the EdwardsSchrödinger equation,

$$
\left(-\frac{l^{2}}{6} \frac{d^{2}}{d z^{2}}-\frac{\beta \Gamma^{\prime}}{4} \frac{\mathrm{e}^{-2 \kappa z}}{z}+\beta h \kappa^{-1} \mathrm{e}^{-\kappa z}\right) \psi(z)=\varepsilon_{0} \psi(z)
$$

where $\varepsilon_{0}$ acts as a Lagrange multiplier to enforce the constraint of the ground state wavefunction normalization. The monomer density is given by $\rho(z)=|\psi(z)|^{2}$. Eq. (8) also describes a quantum particle at its ground state moving under a combined potential of a 1-d screened Coulomb attraction and an almost linear potential. However, the boundary condition expressed by Eq.(15) is different from the hard-wall boundary condition $\left.\psi\right|_{\mathrm{s}}=0$ usually employed for a quantum particle. Instead $\left.\psi\right|_{\mathrm{s}}=$ $\sqrt{\rho_{a}} \neq 0$ for the present problem implies that the steric force felt by the polyelectrolyte from the charged surface should be modified [20]. Setting $\left.\psi\right|_{\mathrm{s}}=0[8,[9,21]$ in the polyelectrolyte adsorption problems in previous studies are not completely correct.

During the adsorption, the rod-like polyelectrolyte tends to lie down on the charged surface. The thickness of the adsorption layer is of the same order of the gyration radius in z-direction. At low ionic strength in which the Debye length is much greater than the layer thickness, the polyelectrolyte cannot feel the potential of length scale much larger than $\kappa^{-1}$, but only the potential barrier height is important. The original potential $V(z)$ in Eq. (8) can thus be replaced by

$$
V_{\text {mod }}(z)= \begin{cases}+\infty, & z<a \\ V(z), & a \leq z<z_{\mathrm{br}} \\ V\left(z_{\mathrm{br}}\right), & z \geq z_{\mathrm{br}}\end{cases}
$$

where $z_{\mathrm{br}}$ is chosen such that $V^{\prime}\left(z_{\mathrm{br}}\right)=0$ and $V\left(z_{\mathrm{br}}\right)$ is the barrier height. In the limit of $\sigma=\kappa=0$, analytic solution gives

$$
\psi(z)=\mathrm{W}_{\lambda, 1 / 2}\left(\frac{3 \beta \Gamma^{\prime}}{2 l^{2} \lambda} z\right) ; \varepsilon_{0}=-\frac{3 \beta^{2} \Gamma^{\prime 2}}{32 l^{2}} \frac{1}{\lambda^{2}}
$$

where $\mathrm{W}_{\lambda, 1 / 2}$ is the Whittaker's notation of the confluent hypergeometric function [22], and $\lambda$ is the least value satisfying the boundary condition. Bound state exists for arbitrary $\epsilon^{\prime} / \epsilon>1$. It implies the threshold surface charge density, $\sigma_{\mathrm{t}}>0$ at low ionic strength.

For both $\sigma, \kappa>0$, no exact solution exists in general but one can analyze it around the transition. Near the surface, the image charge attraction dominates over the surface charge repulsion and hence the binding energy is approximated by the $\sigma=0$ case in Eq. (10). The polyelectrolyte undergoes a de-sorption transition when the binding energy meets the barrier height $V\left(z_{\mathrm{br}}\right)$. After some algebra, we have $\sigma_{\mathrm{t}} \sim\left(\epsilon^{\prime} / \epsilon-1\right)$ for $\epsilon^{\prime} / \epsilon \gg 1$ and $\sigma_{\mathrm{t}} \sim\left(\epsilon^{\prime} / \epsilon-1\right)^{3}$ for $\epsilon^{\prime} / \epsilon \gtrsim 1$. This analytic result is consistent with our simulation data as shown in Fie 2 h.
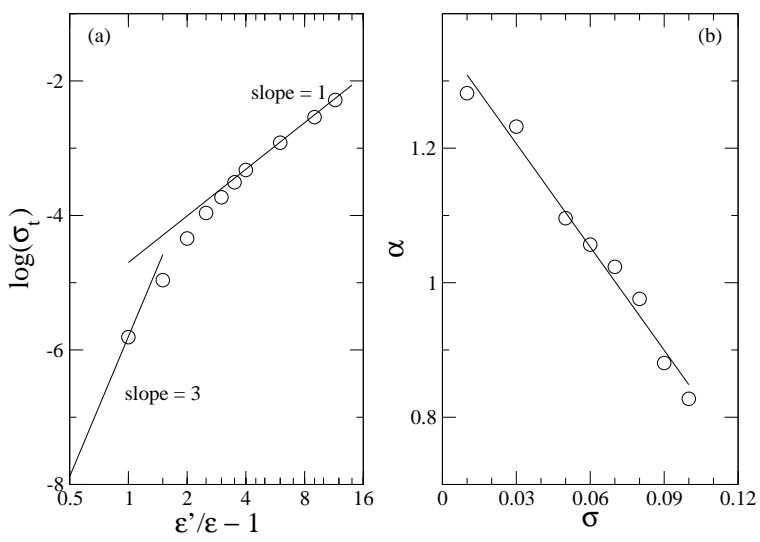

FIG. 2: (a) Simulation results for the surface charge density at the transition, $\sigma_{\mathrm{t}}$, as a function of dielectric ratio $\epsilon^{\prime} / \epsilon$ in logarithmic scale at $\kappa^{-1}=25$. The straight lines indicate slopes of 1 and 3 as suggested in the text. (b) Simulation results for the inverse decay length $\alpha$ (which is proportional to the inverse layer thickness) as a function of $\sigma$ (in unit of $\left.q_{0} / 4 a^{2}\right)$ for $\epsilon^{\prime} / \epsilon=12.5, \kappa^{-1}=25$. The straight line is a linear fit. $\sigma_{\mathrm{t}}=0.102$ in this case. $\alpha$ is obtained from exponential fitting to the tail of corresponding density profile.

An approximate solution for the density profile $\rho(z)$ for the $\sigma>0$ case can be obtained by variational method with trial wavefunction

$$
\psi(z)=\sqrt{\rho_{a}}(1+\mu \alpha(z-a)) \mathrm{e}^{-\frac{1}{2} \alpha(z-a)}
$$

where $\alpha^{-1}$ is the decay length. $\mu$ is positive because the trial wavefunction is restricted to be nodeless. $\alpha$ and $\mu$ are not independent but related via the wavefunction normalization condition. The inverse decay length is calculated to be

$$
\alpha=3 \beta \Gamma^{\prime} / 2 l^{2}+\rho_{a}
$$

where the leading term is independent of $\sigma$. Near the transition, the decay length and hence the thickness of the adsorption layer increase and remain finite. From Eq.(15) and (12), we get the scaling behavior

$$
\alpha-\alpha_{\mathrm{t}} \sim\left(\sigma_{\mathrm{t}}-\sigma\right) \quad \text { for } \quad 0<\sigma<\sigma_{\mathrm{t}}
$$


where $\alpha_{\mathrm{t}}$ is the threshold inverse layer thickness at $\sigma=$ $\sigma_{\mathrm{t}}^{-}$. The variation of $\alpha$ as a function of $\sigma$ obtained from the simulations is shown in Fig 2] which can be well fitted to a linear relation consistent with Eq. (13). On the other hand, for the case of adsorption onto an attractive charged surface $(\sigma<0)$ with substrate of $\epsilon^{\prime} / \epsilon \leq 1$ (e.g. DNA in aqueous solution adsorbed onto a charged lipid membrane), the asymptotic solution to Eq.(8) reproduces the usual scaling $\alpha \sim|\sigma|^{\frac{1}{3}}$ and is a continuous adsorption transition [13], and the thickness swells to infinity as the polyelectrolyte is de-sorbed.

A strongly charged polyelectrolyte immersed in a salt solution will attract oppositely charged ions to condense until its effective charge density reaches the Manning threshold [23]. This means that one can just renormalize $q_{0}$ in our system to $2 e a / l_{\mathrm{B}}$ if $q_{0}$ is larger than $2 e a / l_{\mathrm{B}}\left(l_{\mathrm{B}}\right.$ is the Bjerrum length). Similarly, the strongly charged surface of bare charge density larger than $\kappa /\left(\pi l_{\mathrm{B}}\right)$ is just renormalized back to $\kappa /\left(\pi l_{\mathrm{B}}\right)$ 24]. The Gouy-Chapman length, calculated from the nonlinear Poisson-Boltzmann theory, is of the order $\left(l_{\mathrm{B}} \sigma\right)^{-1}$, which is very large around the transition. However, the nonlinear PoissonBoltzmann potential 25] near the substrate is given by

$$
\phi_{\mathrm{PB}}(z)=\frac{2 l_{\mathrm{B}}}{\epsilon} \ln \frac{1+\gamma \mathrm{e}^{-\kappa z}}{1-\gamma \mathrm{e}^{-\kappa z}}=\phi_{0}-\frac{2 l_{\mathrm{B}} z}{\epsilon \lambda_{\mathrm{GC}}}+O\left(z^{2}\right)
$$

where $\lambda_{\mathrm{GC}}$ is the Gouy-Chapman length and $\gamma=$ $\sqrt{\kappa^{2} \lambda_{\mathrm{GC}}^{2}+1}-\kappa \lambda_{\mathrm{GC}}$. Notice that the linear term in $z$ is proportional to $\lambda_{\mathrm{GC}}^{-1} \propto \sigma$ and is identical to the linear term as expanded from the Debye-Hückel potential. It is not surprising since both Poisson-Boltzmann and Debye-Hückel potentials share the same boundary condition. When the adsorbed polyelectrolyte layer thickness is of one to two monomer size (as seen from $\alpha^{-1} \sim 1$ in Fig.2b), the surface potential felt by the polyelectrolyte should be linear. Physically speaking, it does not matter whether the potential is Poisson-Boltzmann or DebyeHückel, or even the linear one if the layer thickness is much smaller than $\lambda_{\mathrm{GC}}$ and $\kappa^{-1}$. The potential near the surface is determined from the boundary condition. Unlike the case of an attractive surface [26], the effect from large Guoy-Chapman length near the transition is irrelevant in the high-dielectric case.

Our results on the single polyelectrolyte adsorption may provide a starting point to study the charge inversion and multi-layer adsorption 27]. At low ionic strength, polyelectrolytes are adsorbed in a multi-layer structure because of strong Coulomb repulsion. Each layer is composed of parallel 1d Wigner crystal [28]. The upper bound of the multi-layer thickness is $z_{\mathrm{br}} \sim$ $\sigma^{-\frac{1}{2}}\left(\epsilon^{\prime} / \epsilon-1\right)^{\frac{1}{2}}$. It suggests we can easily adjust a single layer adsorbed onto a high-dielectric substrate by tuning the surface charge density. Rigorous treatment based on this physical picture will be elaborated elsewhere.

In conclusion, the adsorption transition of a single polyelectrolyte on a high-dielectric substrate is first or- der since the polyelectrolyte needs to overcome a binding energy from its image charge. Because of the strong Coulomb attraction as compared to the linear repulsive potential near the surface, the polyelectrolyte is compressed without any conformational change before the de-sorption. A scaling law for the adsorption layer thickness is also derived and verified by simulation.

$\mathrm{CHC}$ would like to thank M. Rubinstein for helpful comment. The work is supported by National Science Council of Republic of China under Grant No. NSC922112-M008-051.

* Electronic address: phcch@hotmail.com

[1] P.G. de Gennes, Macromolecules 14, 1637 (1981).

[2] P.G. de Gennes and P. Pincus, J. Phys. Lett. (Paris) 44 L241 (1983).

[3] E. Eisenreigler, K. Kremer, and K. Binder, J. Chem. Phys. 77, 6291 (1982).

[4] P.Y. Lai, Phys. Rev. E 49, 5420 (1994).

[5] W.M. Gelbart, R.F. Bruinsma, P.A. Pincus, and V.A. Parsegian, Physics Today 53, 38 (2000).

[6] Electrostatic Effects in Soft Matter and Biophysics, edited by C. Holm, P. Kékicheff, and R. Podgornik, (Kluwer, 2001).

[7] A.Y. Grosberg, T.T. Nguyen, and B.I. Shklovskii, Rev. Mod. Phys. 74, 329 (2002).

[8] F.W. Wiegel, J. Phys. A 10, 299 (1977).

[9] M. Muthukumar, J. Chem. Phys. 86, 7230 (1987).

[10] R. Varoqui, A. Johner, and A. Elaissari, J. Chem. Phys. 94, 6873 (1991).

[11] I. Borukhov, D. Andelman, and H. Orland, Europhys. Lett. 32, 499 (1995).

[12] X. Chatellier and J.F. Joanny, J. Phys. II 6, 1669 (1996).

[13] J.F. Joanny, Eur. Phys. J. B 9, 117 (1999).

[14] R.R. Netz and J.F. Joanny, Macromolecules 32, 9013 (1999).

[15] F.J. Solis and M.O. de la Cruz, J. Chem. Phys. 110, 11517 (1999)

[16] T.T. Nguyen, A.Y. Grosberg, and B.I. Shklovskii, Phys. Rev. Lett. 85,1568 (2000).

[17] M. Doi and S.F. Edwards, The Theory of Polymer Dynamics, (Oxford Univ., 1986).

[18] T. Garel, H. Orland, and E. Pitard, in Spin Glasses and Random Fields, edited by A.P. Young, (World Sci., 1998).

[19] O.V. Borisov, E.B. Zhulina, and T.M. Birshtein, J. Phys. II France 4, 913 (1994).

[20] C.H. Cheng et.al. (unpublished).

[21] R.R. Netz and D. Andelman, Phys. Rep. 380, 1 (2003).

[22] R. Loudon, Am. J. Phys. 27, 649 (1959).

[23] G.S. Manning, J. Chem. Phys. 51, 924 (1969).

[24] L. Bocquet, E. Trizac, and M. Aubouy, J. Chem. Phys. 117, 8138 (2002).

[25] J.N. Israelachvili, Intermolecular and Surface Forces, (Academic, London, 1985).

[26] A.V. Dobrynin, A. Deshkovski, and M. Rubinstein, Phys. Rev. Lett. 84, 3101 (2000).

[27] A.V. Dobrynin, A. Deshkovski, and M. Rubinstein, Macromolecules 34, 3421 (2001).

[28] B.I. Shklovskii, Phys. Rev. Lett. 82, 3268 (1999). 